\title{
Physiological Effects of Aquaporin in Regulating Drought Tolerance through Overexpressing of Festuca arundinacea Aquaporin Gene FaPIP2;1
}

\author{
Lili Zhuang', Mengxian Liu, Xiuyun Yuan, and Zhimin Yang \\ College of Agro-grassland Science, Nanjing Agriculture University, Nanjing, Jiangsu, 210095, \\ P.R. China \\ Bingru Huang1 \\ Department of Plant Biology and Pathology, Rutgers University, New Brunswick, NJ 08901
}

\begin{abstract}
AdDitional INDEX words. drought stress, tall fescue, water-channel protein, PIP2;1
ABstract. Aquaporin (AQP) proteins serve important roles in regulating water movement across cellular membranes and affect plant responses to drought stress. The objective of this study was to characterize and examine functions of an AQP gene FaPIP2; 1, isolated from a drought-tolerant perennial grass species tall fescue (Festuca arundinacea), for involvement in leaf dehydration status during water stress by overexpressing the gene in arabidopsis (Arabidopsis thaliana). FaPIP2; 1 had characteristic transmembrane domains and Asn-Pro-Ala motifs and was similar to PIP2;1 in rice (Oryza sativa) and maize (Zea mays). Quantitative real-time reverse transcriptase polymerase chain reaction analysis showed that $\mathrm{FaPIP2}$; 1 was upregulated during moderate water stress (hydroponic culture, osmotic potential $\left(\Psi_{S}\right)$ at -0.47 and $\left.-0.78 \mathrm{MPa}\right)$ and the transcript level decreased as $\Psi_{S}$ further decreased. Transgenic arabidopsis plants overexpressing FaPIP2;1 showed greater number of leaves per plant and improved survival rate compared with the wild type (WT) during drought stress. Transgenic plants also maintained higher leaf relative water content (RWC), chlorophyll content (Chl), net photosynthetic rate (Pn), and lower leaf electrolyte leakage (EL) than the WT. However, there was no difference in root length between the transgenic and WT plants following drought stress. The results demonstrated that overexpressing $F$ aPIP 2; 1 could improve plant tolerance to drought stress by enhancing leaf water status, $\mathrm{Chl}$, and photosynthetic rate, as well as maintaining improved cellular membrane stability relative to the WT plants. FaPIP2;1 may be used as a candidate gene for genetic modification of perennial grasses to develop new drought-tolerant germplasm and cultivars.
\end{abstract}

Drought stress caused by lack of rainfall or irrigation is a major abiotic stress limiting plant growth and crop productivity worldwide (Fischer and Turner, 1978). Efficient water transport in plant organs is a critical process to maintain cellular function such that plants can better tolerate dehydration during drought stress. Water transport across plant membranes is controlled by water channel proteins known as AQPs (Gomes et al., 2009; Maurel, 2007; Maurel et al., 2008; Tyerman et al., 2002). Tobacco (Nicotiana tabacum) NtAQP1 is reported to participate in promoting water use efficiency and hydraulic conductivity (Sade et al., 2010). Therefore, understanding and characterizing AQP functions is important to improve plant tolerance to drought stress.

Aquaporins belong to a large family of conserved intrinsic proteins that include five subfamilies with characterized domains and motifs (Gupta and Sankararamakrishnan, 2009; Lian et al., 2004; Suga et al., 2002; Vera-Estrella et al., 2004). A large number of AQP genes have been identified, including 35 in arabidopsis, 36 in maize, and 33 in rice (Chaumont et al., 2001; Johanson et al., 2001; Sakurai et al., 2005). The orthologs are further divided into five types, which mainly include plasma

Received for publication 11 May 2015. Accepted for publication 2 June 2015. We wish to thank Patrick Burgess, David Jespersen, and Jillian Keough at Rutgers University for critical review of the manuscript. This research was supported by the China Postdoctoral Science Foundation Funded Project (no. 2013M541688).

${ }^{1}$ Corresponding author. E-mail: zhuanglili2001@163.com or huang@aesop. rotgers.edu. membrane intrinsic proteins (PIPs), tonoplast intrinsic proteins (TIPs), nodulin 26-like intrinsic proteins (NIPs), small basic intrinsic proteins (SIPs), and X-intrinsic proteins (XIPs) (Danielson and Johanson, 2008; Forrest and Bhave, 2007; Gupta and Sankararamakrishnan, 2009; Heymann and Engel, 1999; Maurel, 2007). Various studies have examined the involvement of AQPs in drought tolerance of different plant species but conflicting results were reported as to which specific mechanisms are responsible for AQP regulation of plant water regulation. Overexpression of TaAQP7, a PIP2 subgroup AQP gene, conferred drought tolerance in transgenic tobacco mainly by promoting water retention and reducing accumulation of reactive oxygen species (ROS) and membrane damages as well as promoting antioxidant metabolism (Zhou et al., 2012a). Heterologous expression of a banana (Musa acuminata) PIP1 subfamily gene, MaPIPI; 1 , in arabidopsis increased salinity and drought tolerance by reducing damage to membranes, promoting ion distribution, and maintaining osmotic equilibrium (Xu et al., 2014). However, heterologous expression of a barley (Hordeum vulgare) AQP gene, HvPIP2; 1, increased leaf water loss in rice (Hanba et al., 2004) and overexpression of NtPIPlb in transgenic tobacco accelerated leaf wilting under drought stress (Aharon, 2003). Varying results as to the relationship between AQP gene expression and whole-plant drought tolerance suggest AQPs regulating physiological processes for conferring drought tolerance may be species specific. Further insight is needed to better describe the mechanisms for AQP regulation of drought tolerance in different plant species. 
Table 1. Accession number of proteins from maize, rice, arabidopsis, and tall fescue for construction of phylogenetic tree.

\begin{tabular}{llcl}
\hline Protein name & Accession no. & Protein name & Accession no. \\
\hline ZmPIP1;1 & CAA57955.1 & OsPIP1;1 & BAD28398.1 \\
ZmPIP1;2 & AAD29676 & OsPIP1;2 & Q7XSQ9.3 \\
ZmPIP1;3 & AAK26754.1 & OsPIP1;3 & BAD22920.1 \\
ZmPIP1;4 & AAK26755.1 & OsPIP2;1 & BAC15868.1 \\
ZmPIP1;5 & AAK26756.1 & OsPIP2;2 & BAD23735.1 \\
ZmPIP1;6 & AAK26757.1 & OsPIP2;3 & CAD41442.1 \\
ZmPIP2;1 & AAK26758.1 & OsPIP2;4 & BAC16113.1 \\
ZmPIP2;2 & AAK26759.1 & OsPIP2;5 & BAC16116 \\
ZmPIP2;3 & AAK26760.1 & OsPIP2;6 & CAE05002.2 \\
ZmPIP2;4 & AAK26761.1 & OsPIP2;7 & BAD46581.1 \\
ZmPIP2;5 & AAD28761.1 & OsPIP2;8 & AAP44741.1 \\
ZmPIP2;6 & AAK26762.1 & - & - \\
ZmPIP2;7 & AAK26763.1 & - & - \\
AtPIP1;1 & CAB71073 & FaPIP2;1 & KP258181 \\
AtPIP1;2 & AAC28529 & - & - \\
AtPIP1;3 & AAF811320 & - & - \\
AtPIP1;4 & AAF02782 & - & - \\
AtPIP1;5 & CAA20461 & - & - \\
AtPIP2;1 & CAB67649 & - & - \\
AtPIP2;2 & AAD18142 & - & - \\
AtPIP2;3 & AAD18141 & - & - \\
AtPIP2;4 & BAB09839 & - & - \\
AtPIP2;5 & CAB41102 & - & - \\
AtPIP2;6 & AAC79629 & - & - \\
AtPIP2;7 & CAA17774 & - & - \\
AtPIP2;8 & AAC64216 & - & - \\
\hline
\end{tabular}

AQP genes have been isolated and characterized in the model species arabidopsis and also in agronomic crops such as rice and maize. There are few AQP genes that have been isolated or cloned from perennial grass species except for a PIP2homologue shown to have salinity-induced expression in Festuca rubra ssp. litoralis (Diedhiou et al., 2009). Understanding the molecular mechanisms by which perennial grasses survive under prolonged drought conditions may be beneficial to genetic improvement of stress tolerance in grass species (Fry and Huang, 2004; Zhou et al., 2012b). Unfortunately, there exists little knowledge about AQPs in perennial grass species because of lack of complete genomic sequences and allopolyploid nature. Furthermore, there is a lack of knowledge regarding the physiological roles of AQPs in drought stress tolerance. Therefore, the objective of this study was to determine the function of AQP gene FaPIP 2;1 from tall fescue in plant tolerance to drought stress by overexpressing the gene in arabidopsis. Tall fescue used as turfgrass is known to be better adaptive to drought stress than many other cool-season turfgrass species (Fry and Huang, 2004). Confirmation of FaPIP2; 1 functions in regulating drought tolerance using the model plant species is important for determining the utility of this gene for future work in genetic modification of perennial grasses to develop new drought-tolerant germplasm and cultivars.

\section{Materials and Methods}

Plant materials, growth conditions, and stress TREATMENTS. Tillers of tall fescue (cv. Barlexas) were collected from mature stock plants and transferred into half-strength
Hoagland's nutrient solution (Hoagland and Arnon, 1950) with a nonstress $\Psi_{\mathrm{S}}$ of $-0.19 \mathrm{MPa}$. Plants were wrapped in a piece of sponge, which were inserted in holes on a Styrofoam plate floating on the nutrient solution in a plastic container $(40 \times 30$ $\times 15 \mathrm{~cm})$. Nutrient solution was aerated by aquarium air pump (LifeTech, Shenzhen, China). Plants were grown for $30 \mathrm{~d}$ to develop new roots and shoots after which time polyethylene glycol (PEG 6000; Huada, Shantou, China) was added to the growing solution every $5 \mathrm{~d}$ to incrementally reduce $\Psi_{\mathrm{S}}$ to $-0.47,-0.78,-1.22$, and $-1.61 \mathrm{MPa}$ inducing and increasing the level of drought stress. Each PEG treatment and the control treatment had four replicates (four plastic containers) with multiple plants within each replicated container. Vapor pressure osmometer (Wescor, Logan, UT) was used to monitor the $\Psi_{\mathrm{S}}$ of growing solution as described by Michel (1983). Plants were maintained in a controlled-environment growth chamber (MT8070iE; Xubang, Henan, China) set to $12 \mathrm{~h}$ photoperiod, $70 \%$ relative humidity, $500 \mu \mathrm{mol} \cdot \mathrm{m}^{-2} \cdot \mathrm{s}^{-1}$ photosynthetically active radiation $(P A R)$, and a day/night temperature of $25 / 15^{\circ} \mathrm{C}$.

ISOLATION AND CLONING OF FULL-LENGTH CDNA ENCODING FAPIP2;1. Leaves were collected from tall fescue plants grown in the control nutrient solution for the isolation of the AQP genes (designated as FaPIP 2;1). Partial sequence of FaPIP2; 1 was obtained from tall fescue expressed sequence tags (ESTs) (Mian et al., 2008) through the Local Blast program in BioEdit software (Hall, 1999). Nested PCR was performed using primers F1 (5'-GATGGCGAAGGACGAGGT (G/T) GT-3') and F2 (5' -CTTCGGCGGCATGATCTTCG-3') paired with adaptor R (5' -CTGATCTAGAGGTACCGGATCCATAT-3'). The polymerase chain reaction (PCR) conditions were set as initial denaturation at $94{ }^{\circ} \mathrm{C}$ for $5 \mathrm{~min}$, then 30 cycles of $94{ }^{\circ} \mathrm{C}$ for $3 \mathrm{~min}, 58^{\circ} \mathrm{C}$ for $30 \mathrm{~s}$, and $72^{\circ} \mathrm{C}$ for $1 \mathrm{~min}$. The PCR product was inserted into T-Vector pMD ${ }^{\mathrm{TM}} 19$ (Simple) (Takara, Otsu, Japan) and sequenced. The full-length coding sequence was obtained with gene-specific primers based on the blast sequences and 3 '-end sequences. Full-length cDNA encoding FaPIP2;1 was analyzed by DNAMAN 6.0 software (Lynnon Biosoft, Pointe-Claire, Canada) and BLASTX program against the non-redundant protein database at the National Center for Biotechnology Information [NCBI (Gish and States, 1993)].

ChaRACTERIZATION OF FAPIP2; 1 EXPRESSION IN RESPONSE TO DROUGHT STRESS. FaPIP $2 ; 1$ expression in tall fescue responding to drought stress was examined using quantitative real-time reverse transcriptase PCR (qRT-PCR) analysis. Leaf samples were collected at $5 \mathrm{~d}$ PEG treatment for each level of $\Psi_{\mathrm{S}}$ or drought stress, frozen in liquid nitrogen, and stored at $-80{ }^{\circ} \mathrm{C}$. Total RNA was extracted using a Tripure Isolation Reagent Kit (Roche Diagnostic, Basel, Switzerland) according to the manufacture's protocol. RNA integrity was confirmed by electrophoresis and absorbance at 260/280 nm. First-strand cDNA was obtained by using PrimeScript RT Reagent Kit (Takara). gDNA was removed by gDNA Eraser at $42{ }^{\circ} \mathrm{C}$ for 2 min according to manufacturers' instruction by using PrimeScript RT Reagent Kit with gDNA Eraser (Takara). The qRTPCR was performed on LightCycler480II machine (Roche Diagnostic) with SYBR Green I Master (Roche Diagnostic). The PCR condition was as follows: initial denaturation step of 10 min at $95^{\circ} \mathrm{C}$ followed by 40 cycles of PCR $\left(95^{\circ} \mathrm{C}\right.$ for $15 \mathrm{~s}$, $60{ }^{\circ} \mathrm{C}$ for $15 \mathrm{~s}$, and $72{ }^{\circ} \mathrm{C}$ for $\left.30 \mathrm{~s}\right)$. Data were collected at $65^{\circ} \mathrm{C}$ in each cycle. Primers for qRT-PCR were FaPIP2-1F ( $5^{\prime}$ CCTTCTACCACCAGTACATCCT-3') and FaPIP2-1R 


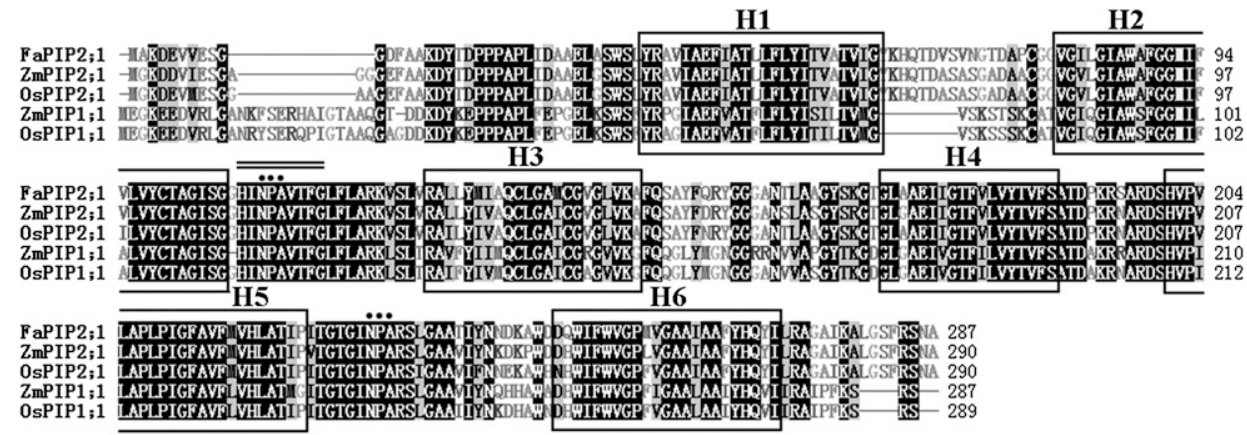

Fig. 1. Alignment of the amino acid sequence of FaPIP2;1 with other PIP proteins. Six transmembrane helixes designated as $\mathrm{H} 1-\mathrm{H} 6$ are shown in the rectangle. The most conserved amino acid sequences "HINPAVTFG" were marked with double transverse lines. Black dots indicated "NPA" motif. The figure was constructed by Cluster X program in BioEdit software (Hall, 1999). The accession numbers of the proteins used are as follow: FaPIP2;1 (KP258181), ZmPIP2;1 (AAK26758.1), OsPIP2;1 (BAC15868.1), ZmPIP1;1 (CAA57955.1), and OsPIP1;1 (BAD28398.1).



Fig. 2. Phylogenetic tree of PIP proteins from tall fescue, maize, rice, and arabidopsis. FaPIP2;1 was labeled with a black dot. The corresponding accession numbers of the amino acid sequence are indicated in Table 1 . The phylogenetic tree was constructed based on consensus amino acid sequence by MEGA4.0 software (Tamura et al., 2007). Branch lengths indicate distance.

(5'-GCTTCAGCACACTCATCATCTT-3'). FaActin was used as reference gene, and the primers used were FaActinF $\left(5^{\prime}\right.$-GCTCAACCCCAAGGCTAAC-3') and FaActinR (5'AGAGCGTATCCCTCGTAGATG-3'). Three technical and biological replicates were conducted. The relative expression level of FaPIP 2; 1 was calculated by $2^{-\triangle \Delta C T}$ method.

TRANSFORMATION AND GENERATION OF ARABIDOPSIS PLANTS OVEREXPRESSING $F_{A} P I P 2 ; 1$. The recombinant plasmid pEarleyGate103-FaPIP2;1-GFP was transferred into Agrobacterium tumefaciens strain EHA105. Transgenic arabidopsis plants were obtained by Agrobacterium-mediated floral dip method based on the ecotype 'Columbia' (Col-0) (Clough and Bent, 1998). $\mathrm{T}_{1}$ generation transgenic plants were selected on Murashige Skoog (MS) media petri dishes supplemented with $20 \mathrm{mg} \cdot \mathrm{L}^{-1}$ glufosinateammonium [GLA (Sigma, Shanghai, China)]. The $\mathrm{T}_{1}$ transgenic seedlings were verified by PCR method using forward primer specific to pEarleyGate103 (5' -TATCCTTCGCAAGACCCTTCCTCTA- $3^{\prime}$ ) and reverse primer specific to FaPIP2;1 (5'-GATATCTGGCGTTGCTCCT GAAGGAGCC- $3^{\prime}$ ). Seeds of the $T_{2}$ generation were collected and germination rate was calculated on MS media petri dishes (supplemented with $20 \mathrm{mg} \cdot \mathrm{L}^{-1} \mathrm{GLA}$ ). Two transgenic lines (13 and 18) that survived on MS media containing $20 \mathrm{mg} \cdot \mathrm{L}^{-1}$ GLA were selected for further analysis.

Transformation of FaPIP2;1 expression level was confirmed using RT-PCR analysis in transgenic lines of arabidopsis. RNA was extracted from arabidopsis leaves as mentioned above and first-strand cDNA synthesis was performed with a Transcriptor One-step RT-PCR Kit (Roche Diagnostic) using $0.5 \mu \mathrm{g}$ RNA as template. The primer for RT-PCR was RTFaPIP2-1F (5' -CTTCCAGAGCGCCTACTTTCAG-3') and RTFaPIP2-1R (5' -ATCCCATGCCTTGTCATTGTTG-3'). AtACTIN2 was used as internal control and primers used were AtActin2F (5'-CCTTGAAGTATCCTATTGAGC-3') and AtActin2R (5'-GGTCTTTGAGGTTTCCATCT-3').

GROWTH AND PHYSIOLOGICAL EVALUATION OF DROUGHT TOLERANCE FOR WILD TYPE AND TRANSGENIC ARABIDOPSIS PLANTS OVEREXPRESSING $\boldsymbol{F}_{A} \boldsymbol{P I P}$; 1 1. Seeds of WT and transgenic plants were sterilized in $1.3 \% \mathrm{NaClO}$ for $8 \mathrm{~min}$, washed six times in distilled-deionized water, and then placed on MS media petri dishes (by 10- $\mu \mathrm{L}$ Eppendorf pipette) and germinated after $3 \mathrm{~d}$ at $4{ }^{\circ} \mathrm{C}$. Seedlings were established in a growth chamber (Haier, Qingdao, China) set at $22^{\circ} \mathrm{C}, 120 \mu \mathrm{mol} \cdot \mathrm{m}^{-2} \cdot \mathrm{s}^{-1}$ light intensity, 16 -h photoperiod, and $70 \%$ relative humidity.

To determine whether overexpressing FaPIP2;1 affected root growth during drought stress, seedlings (7-d old) were maintained either on MS media petri dishes (control) or transferred to MS media petri dishes supplemented with 300 mm mannitol to induce drought stress. After $4 \mathrm{~d}$ treatment, root images were recorded by single-lens reflex camera (D5100; Nikon, Bangkok, Thailand). Seedlings incubated on MS + $300 \mathrm{~mm}$ mannitol were then transferred to MS media without mannitol to recover for $4 \mathrm{~d}$, and root length was measured thereafter. Both the control and mannitol treatments were repeated in three petri dishes with six plants as subsamples within each replicate for the WT and each transgenic line.

Shoot growth and physiological responses of WT and transgenic plants to drought stress was evaluated by transferring 10-d-old seedlings to plastic pots $(9 \mathrm{~cm}$ in diameter and $8.5 \mathrm{~cm}$ in height) filled with sphagnum moss peat (Pindstrup, Ryomgaard, Denmark) and vermiculite $[1: 1(\mathrm{v} / \mathrm{v})]$ and maintained in a growth chamber (Haier) at $22^{\circ} \mathrm{C}$ for $14 \mathrm{~d}$. Irrigation was withheld for an additional $14 \mathrm{~d}$ to impose drought stress while control plants were maintained well watered. Each 


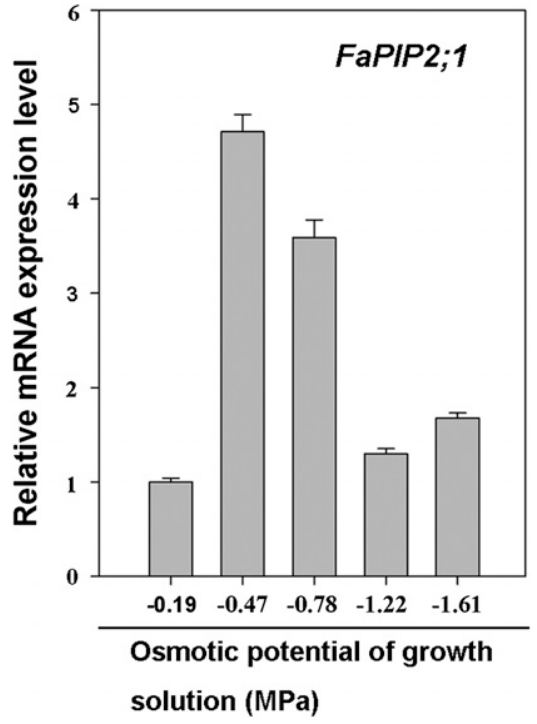

Fig. 3. Relative mRNA expression level of FaPIP2; 1 by quantitative real-time reverse transcriptase polymerase chain reaction (qRT-PCR) in tall fescue under polyethylene glycol (PEG) treatment. The $\Psi_{\mathrm{S}}$ of the growth solution was gradually reduced by adding PEG6000 from $-0.19 \mathrm{MPa}$ in the beginning to an ultimate -1.61 $\mathrm{MPa}$ as shown in the vertical axis. FaActin was used as internal control. Three independent experiments showed similar results, and data from one experiment are presented here. Values are means \pm SD of three technical repetitions. treatment and plant line was replicated four times in four pots with four plants in each pot. Relative leaf number, leaf RWC, $\mathrm{EL}, \mathrm{Chl}$, and Pn were measured at $14 \mathrm{~d}$ of drought stress as described below. Plants were then rewatered and grown for an additional $20 \mathrm{~d}$ to evaluate survival rate.

Leaf number per plant was counted in eight WT and eight transgenic plants in each of four replicates (pots). The ratio of leaf number in transgenic lines to that of the WT was calculated as relative leaf number.

RWC was determined to evaluate leaf hydration status. For leaf $\mathrm{RWC}$ analysis, $\approx 0.1 \mathrm{~g}$ fully expanded leaves from each pot were collected and weighed to obtain fresh weight $(\mathrm{FW})$. Leaves were then immersed into deionized water for $12 \mathrm{~h}$ at $4{ }^{\circ} \mathrm{C}$, removed from water and blotted dry, turgid weight (TW) was obtained. Finally, dry weight (DW) was obtained after leaves were dried thoroughly in an oven at $80^{\circ} \mathrm{C}$. RWC was calculated as follows: RWC $=$ $(\mathrm{FW}-\mathrm{DW}) /(\mathrm{TW}-\mathrm{DW}) \times 100$ (Barrs and Weatherley, 1962).

Leaf EL was used as an indicator of cellular membrane stability (Blum and Ebercon, 1981). For EL analysis, $\approx 0.1 \mathrm{~g}$ fully expanded leaves from plant top in each pot were collected and incubated in $30 \mathrm{~mL}$ distilled-deionized water. The initial level of conductance of the incubation solution $\left(C_{\mathrm{i}}\right)$ was measured after shaken for $24 \mathrm{~h}$ at room temperature using a conductance meter (Orion Star A212 benchtop conductivity meter; Thermo Fisher Scientific, Waltham, MA). Leaf tissue
A

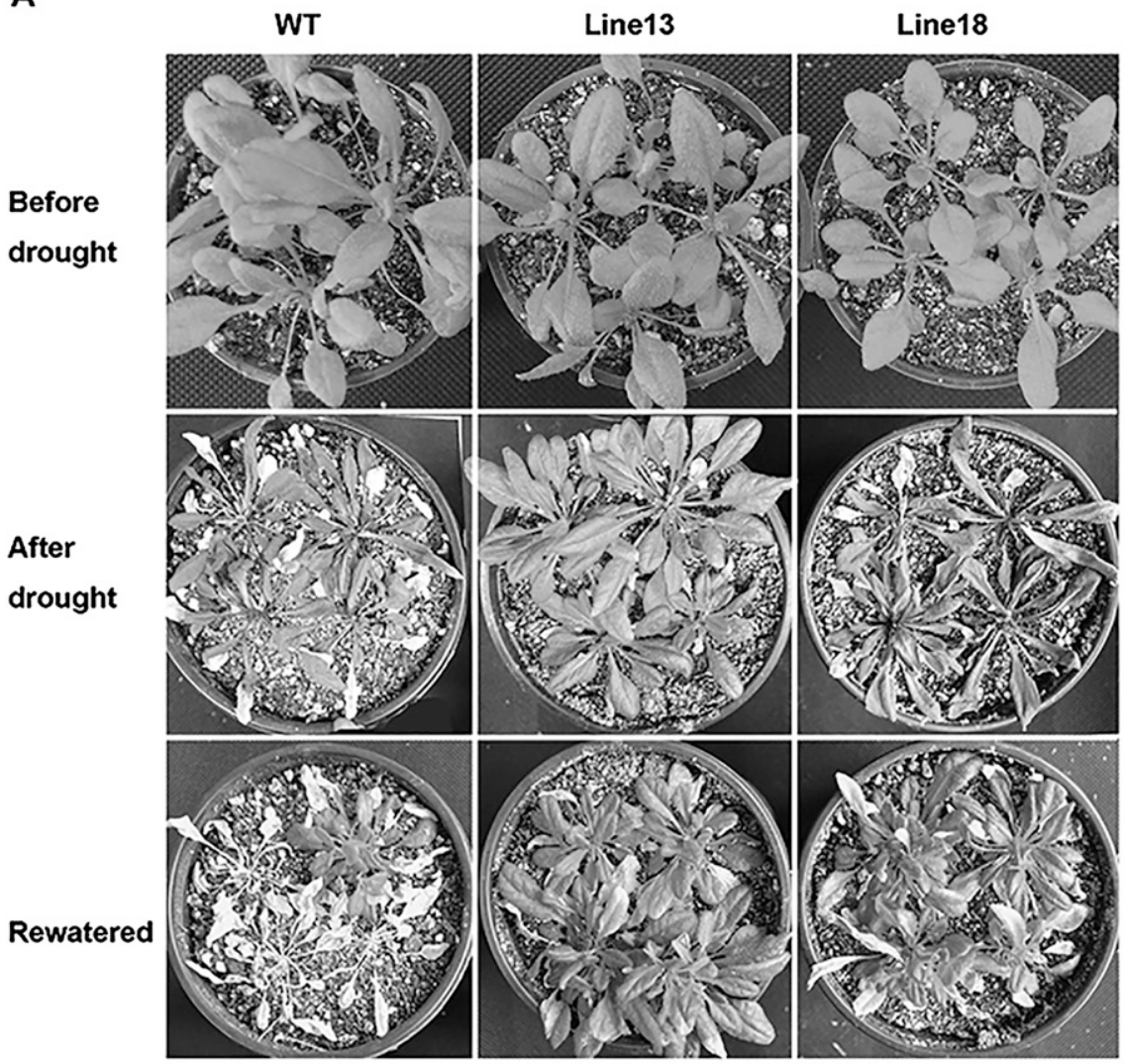

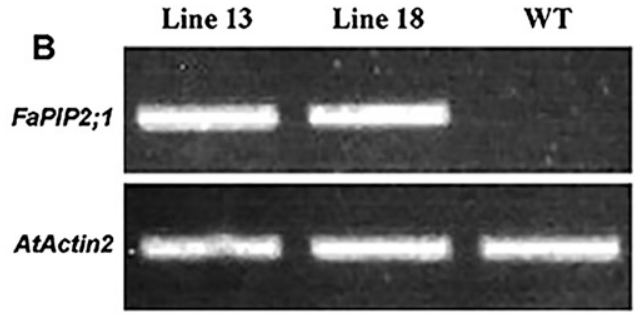

C
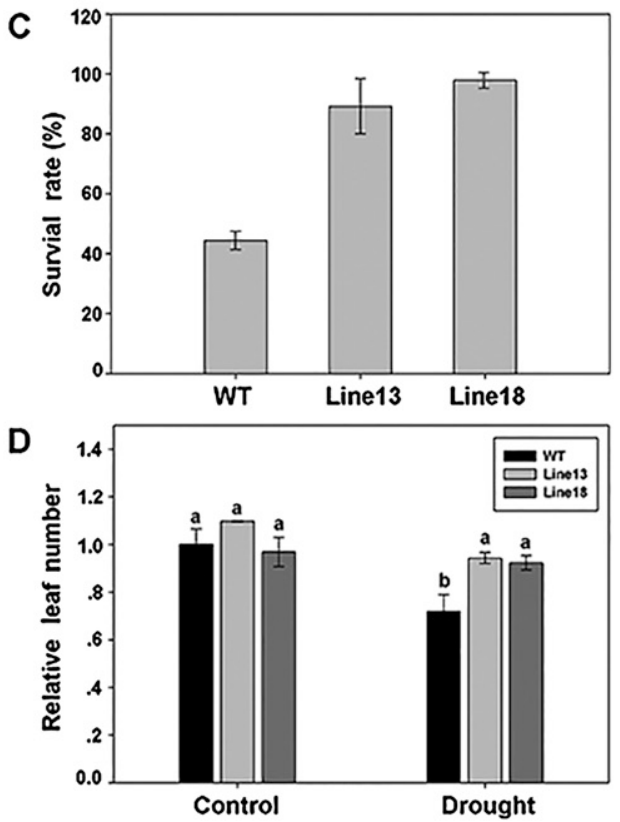

Fig. 4. Expression level and phenotypic characteristic of wild type (WT) and transgenic plants under drought stress. (A) Phenotypic comparison of WT and transgenic plants due to withholding water for $14 \mathrm{~d}$ and rewatered for $20 \mathrm{~d}$. (B) Semiquantitative real-time reverse transcriptase polymerase chain reaction (RTPCR) analysis in WT and two transgenic lines. (C) Survival rate of WT and transgenic lines under drought stress. Values are means \pm SD of 12 independent plants. (D) Relative leaf number of transgenic plants compared with WT under normal and drought conditions. Different letters atop bars indicate significant differences $(P \leq 0.05)$ between WT and transgenic lines under same growth condition. Values are means \pm SD of eight independent plants. 
was then autoclaved at $121^{\circ} \mathrm{C}$ for $30 \mathrm{~min}$ and again shaken for $24 \mathrm{~h}$. Conductance of the solution was determined as $C_{\max }$. Relative $\mathrm{EL}=\left(C_{\mathrm{i}} / C_{\max }\right) \times 100($ DaCosta et al., 2004) .

Leaf Chl was measured to evaluate level of leaf senescence. Leaves were collected and chlorophyll extracted in darkness thoroughly ( $\approx 72 \mathrm{~h}$ ) in $95 \%$ ethyl alcohol. The absorbance of the solution with leaf extract was measured at 663 and $645 \mathrm{~nm}$ by a spectrophotometer (Ultrospec 9000; GE Healthcare Life Sciences, Cambridge, UK). Dry weight was recorded after $72 \mathrm{~h}$ in $80^{\circ} \mathrm{C}$ oven. Chl was calculated as described by Arnon (1949).

Pn was measured on five individual leaves per replicate per treatment using an infrared gas exchange analyzer (LI-6400; LI-COR, Lincoln, NE) with a leaf chamber $\left(6 \mathrm{~cm}^{2}\right)$ supplemented with light-emitting diodes light intensity of 500 $\mu \mathrm{mol} \cdot \mathrm{m}^{-2} \cdot \mathrm{s}^{-1} P A R$ and $400 \mu \mathrm{L} \cdot \mathrm{L}^{-1} \mathrm{CO}_{2}$. Leaves were scanned on a digital scanner and leaf area was determined using an imaging analysis program (Digimizer; MedCalc Software, Mariakerke, Belgium) immediately after Pn measurements.

Statistical analysis. Growth and physiological data were subjected to the analysis of variance using the general linear model of statistics (SAS version 9.0; SAS Institute, Cary, NC). Means were separated by the Fisher's least significance test at $P<0.05$.

\section{Results}

Cloning and Sequence analysis of $\boldsymbol{F}_{\boldsymbol{A}} \boldsymbol{P I P 2}$;1. A 630-bp partial sequence with high similarity to $O S P I P 2 ; 1$ was identified from a tall fescue EST database (Mian et al., 2008) and sequence alignment showed that the $3^{\prime}$ end was missing. Therefore, 3' rapid amplification of cDNA ends (RACE) PCR was performed using cDNA transcribed from mRNA isolated from tall fescue leaves. A full-length 1197-bp cDNA sequence including a 864bp open reading frame (ORF) was obtained and named as FaPIP2;1 (NCBI accession number: KP258181). FaPIP2;1 encoded an AQP protein with 287 amino acids with a predicted molecular mass of $30.2 \mathrm{kDa}$. BlastX analysis revealed that FaPIP 2;1 showed 90\% similarity with OsPIP 2;1 from rice, 90\% similarity with $H v P I P 2 ; 1$ from barley, and $85 \%$ similarity with $T a A Q P 7$ from tobacco. Full-length amino acid sequence alignment showed that FaPIP2;1 had six transmembrane structures and two Asn-Pro-Ala (NPA) motifs (Fig. 1). Phylogenetic analysis indicated that FaPIP2;1 is closely related to OsPIP2;1, which itself is a member of the PIP2 subfamily (Fig. 2).

EXPRESSION PATTERN OF FAPIP2; 1 IN RESPONSE TO DROUGHT STRESS. To determine responses of FaPIP2;1 expression to drought stress, the transcript level of FaPIP2; 1 was measured in leaves of plants exposed to different levels of drought stress induced by different concentrations of PEG with $\Psi_{\mathrm{S}}$ ranging from -0.47 (mild water stress) to $-1.61 \mathrm{MPa}$ (severe water stress). The transcript level of FaPIP 2;1 was significantly higher for all levels of drought stress compared with nonstress control level $\left(-0.19 \mathrm{MPa} \Psi_{\mathrm{S}}\right.$ of nutrient solution without PEG) in response to $\mathrm{PEG}$-induced drought stress, reaching to the maximum level at $-0.47 \mathrm{MPa}$ of $\Psi_{\mathrm{S}}$ of the nutrient solution with PEG; the transcript level was significantly higher at all PEG treatments than that of the nonstress control (Fig. 3).

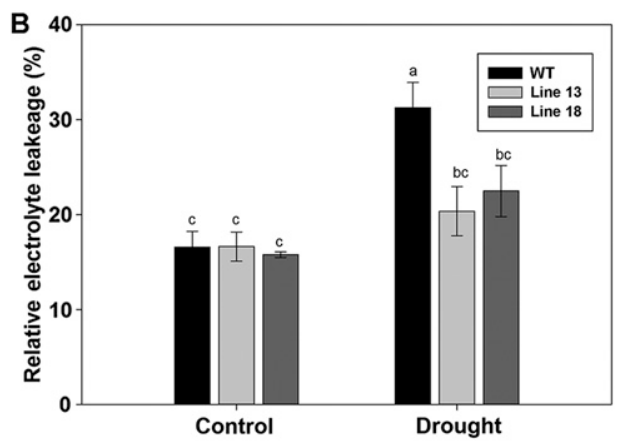

Fig. 5. Physiological analysis in wild type (WT) and transgenic plants under normal and drought conditions. (A) Leaf relative water content (RWC) of WT and transgenic plants under normal and drought conditions. (B) Electrolyte leakage (EL) in WT and transgenic plants under normal and drought conditions. Different letters atop bars indicate significant differences $(P \leq 0.05)$ between WT and transgenic lines under same growth condition. Values are means $\pm \mathrm{SD}$ of four independent repetitions.
IMPROVED LEAF GROWTH, PHYSIOLOGICAL ACTIVITIES, AND POST-DROUGHT RECOVERY OF TRANSGENIC ARABIDOPSIS PLANTS OVEREXPRESSING $F_{A} P I P 2 ; 1$. Transformation of model plants arabidopsis overexpressing FaPIP2;1 was conducted to determine whether FaPIP2; 1 is involved in regulating plant tolerance to drought stress. A total of 34 transgenic lines $\left(T_{1}\right)$ were selected by resistance to GLA and confirmed by PCR using primers specific to FaPIP2;1 and pEarleyGate103
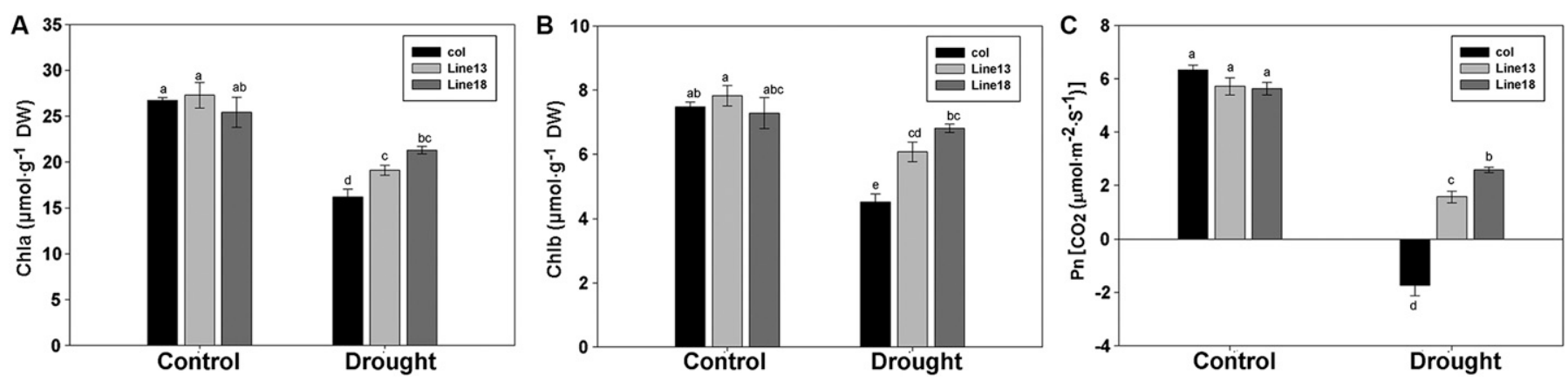

Fig. 6. Analysis of leaf chlorophyll content (Chl) and photosynthetic rate (Pn) in wild type (WT) and transgenic plants under normal and drought conditions. (A) Analysis of Chla content in WT and transgenic plants under normal and drought conditions. (B) Analysis of Chlb content in WT and transgenic plants under normal and drought conditions. (C) Analysis of Pn in WT and transgenic plants under normal and drought conditions. Different letters atop bars indicate significant differences $(P \leq 0.05)$ between WT and transgenic lines under same growth condition. Values are means \pm SD of four independent repetitions. 
(data not shown). Seeds of the $\mathrm{T}_{2}$ generation were collected and two representative $T_{2}$ transgenic lines (13 and 18) were used to compare with the WT in the physiological evaluation of drought tolerance (Fig. 4A). RT-PCR showed that FaPIP2;1 was highly expressed in the transgenic lines but not in WT (Fig. 4B).

Phenotypes of transgenic lines were compared with WT after 14-d drought stress. Leaves of transgenic plants appeared to be more hydrated or turgid than the WT, which exhibited more severe wilting and chlorosis at $14 \mathrm{~d}$ of drought stress (Fig. 4A). Survival rate of plants following drought stress was calculated at $20 \mathrm{~d}$ of rewatering. Most of WT plants were dead with only $37 \%$ survival rate while transgenic plants of line 13 and line 18 had $100 \%$ survival rate (Fig. 4C). Transgenic lines 13 and 18 produced more leaves per plant than the WT during $14 \mathrm{~d}$ of drought stress (Fig. 4A and D).

Under well-watered condition, there were no significant differences between WT and transgenic plants in either leaf RWC (Fig. 5A) or EL (Fig. 5B). Transgenic plants of both lines 13 and 18 had significantly higher RWC [above 60\% (Fig. 5A)] and lower EL [about 20\% (Fig. 5B)] at $14 \mathrm{~d}$ of drought stress.

Transgenic plants did not show significant differences in the content of Chla and Chlb, and Pn from the WT under wellwatered conditions, but exhibited significantly greater content of both Chla (Fig. 6A) and Chlb (Fig. 6B), as well as higher Pn (Fig. 6C) than the WT at $14 \mathrm{~d}$ of drought stress.

ROOT GROWTH OF FAPIP2;1-TRANSGENIC ARABIDOPSIS PLANTS. Total root length per plant showed no difference in both the WT and transgenic plants (Figs. 7A and 8A) on MS media petri dishes, while it decreased significantly with drought stress induced by exposing roots to mannitol (Figs. 7B and 8A). The decrease in total root length was mainly due to the inhibition of root elongation by osmotic stress. Furthermore, transgenic plants from Fig. 7B did not show significant difference in total root length from the WT under either nonstress (Figs. 7C and 8B) or drought stress conditions (Figs. 7D and 8B). The WT and transgenic plants also did not have difference in root number and elongation of tap roots or lateral roots (Fig. 7).

\section{Discussion}

In this study, an AQP gene FaPIP2;1 was cloned and characterized from tall fescue, a species known to exhibit superior drought tolerance as compared with other cool-season grass species (Fry and Huang, 2004). The high degree of amino acid sequence similarity with AQPs from rice and maize, particularly OsPIP 2;1 suggested that FaPIP 2;1 was a conserved AQP gene in grass species. Sequence analysis showed that FaPIP2; 1 had six putative transmembrane $\alpha$-helices and other conserved motifs which are found in all PIP proteins that are known to play vital roles in water channel activity ( $\mathrm{Li}$ et al., 2009).

AQP genes are responsive to water stress but exhibit differential patterns of expression in different plant species. For example, the transcription level of PIP 1;5, PIP 2;2, PIP 2;3, and $P I P 2 ; 6$ decreased to only $10 \%$ of the nonstress level during drought stress in a study with arabidopsis (Jang et al., 2004). However, transcription level of AtPIP1-4 and AtPIP2-5 in arabidopsis leaves increased under drought stress in another study (Alexandersson et al., 2005). The expression of PIP genes like arabidopsis $R D 28$ and the NeMip2 and NeMip3 from Nicotiana excelsior, were upregulated when subjected to
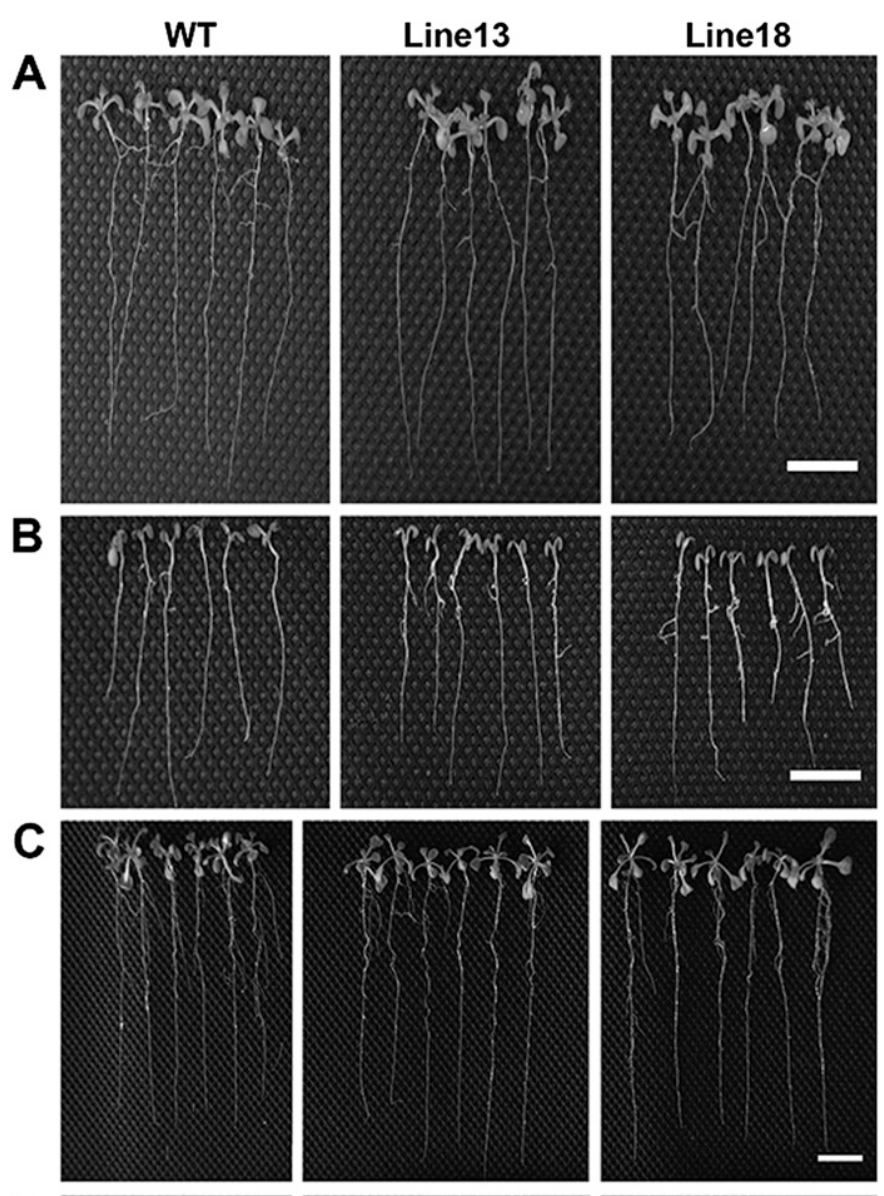

D
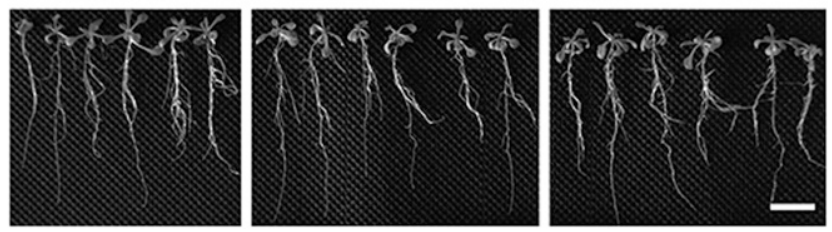

Fig. 7. Root growth responses to drought stress and rewatering of overexpressing FaPIP 2; 1 arabidopsis plants. (A) Root of wild type and transgenic plants grown on Murashige Skoog (MS) media for $4 \mathrm{~d}$. (B) Root of wild type and transgenic plants grown on MS media supplemented with $300 \mathrm{~mm}$ mannitol for 4 d. (C) Root of wild type and transgenic plants grown on MS media for $8 \mathrm{~d}$. (D) Root of wild type and transgenic plants in (B) retransferred to MS media for another $4 \mathrm{~d}$; bars $=1 \mathrm{~cm}$.

drought stress (Yamada et al., 1997; Yamaguchi-Shinozaki et al., 1992). In addition, AQPs isolated from different cultivars of the same species could also respond differently to abiotic stress (Lian et al., 2006). For example, in upland rice, expression level of genes such as OsPIP1;2, OsPIP1;3 (in roots and leaves) and $O s P I P 2 ; 1, O s P I P 2 ; 5$ (in roots) were significantly upregulated, whereas in lowland rice, the corresponding mRNA expression level remained unchanged or downregulated (Lian et al., 2006). In this study, FaPIP2;1 isolated from drought-tolerant tall fescue was significantly upregulated by drought stress to the highest level under mild drought stress $(-0.47 \mathrm{MPa})$ suggesting that FaPIP $2 ; 1$ could play positive roles in regulating plant adaptation to water stress.

Although AQPs are widely recognized for their functions in assisting with transmembrane water transport, their effects on plant tolerance to abiotic stresses (i.e., drought stress) are inconsistent across different plant species. Some studies 

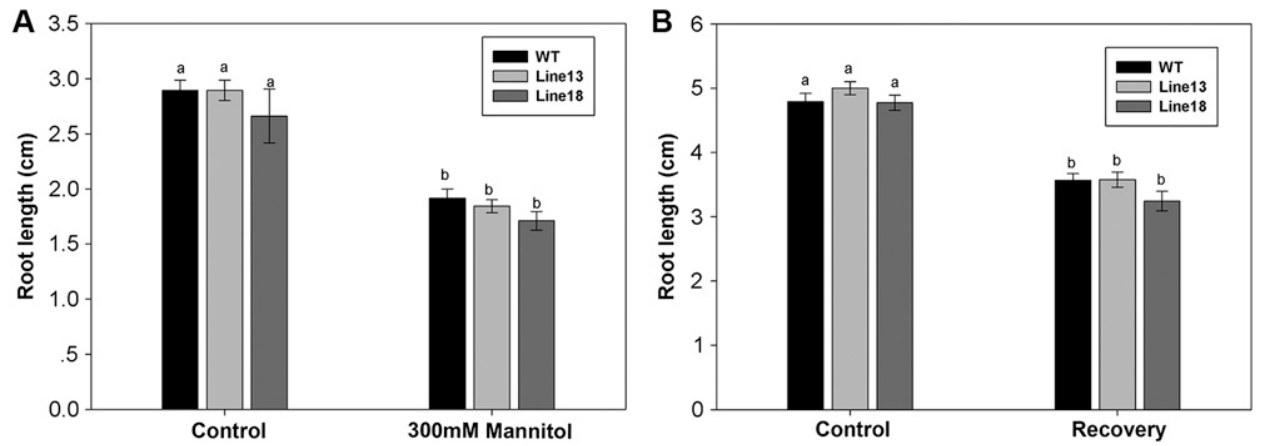

Fig. 8. Primary root length of wild type (WT) and transgenic lines under control, drought, and recovery conditions. (A) Primary root length of WT and transgenic lines under normal [Murashige Skoog (MS) media] or drought (MS media supplemented with $300 \mathrm{~mm}$ mannitol) for $4 \mathrm{~d}$. (B) Primary root length of WT and transgenic lines under normal (grown on MS media for $8 \mathrm{~d}$ ) or recover condition (first on MS media supplemented with $300 \mathrm{~mm}$ mannitol for $4 \mathrm{~d}$, then transferred to MS media for another $4 \mathrm{~d}$ ). Data are means \pm SE of $n=18$ biological replicates. Means marked with the same letter showed no significant difference at $P<0.05$ as calculated by Duncan's multiple range test.

reported positive effects of AQP gene expression on improving plant stress tolerance to salinity, drought, and chilling stress (Aroca et al., 2005; Cui et al., 2008; Gao et al., 2010; Guo et al., 2006; Johanson et al., 2001; Mahdieh et al., 2008; Yu et al., 2006), whereas others reported decreases or no effects of overexpressing AQP genes on stress tolerance (Aharon, 2003; Wei et al., 2006). The discrepancy in roles of AQP genes for regulating stress tolerance in different studies has been attributed to different experimental conditions and to the variations of genes isolated from different plant species (Aharon, 2003).

To further understand the function of FaPIP2;1 in the regulation of drought tolerance, transgenic arabidopsis plants overexpressing FaPIP2;1 were generated and several of the most commonly used physiological indicators for drought tolerance, including RWC, EL, Chl, and Pn were examined in this study. The enhanced leaf RWC in FaPIP2; 1-transgenic plants under drought stress suggested that FaPIP2;1 could be involved in maintaining cellular hydration of leaves. Overexpression of some AQP genes such as $R W C 3$ in rice and NtAQP1 in tobacco, have been reported to serve important roles in promoting water-use efficiency, hydraulic conductivity, and maintaining water status of plants under drought stress (Cui et al., 2008; Li et al., 2008; Lian et al., 2004; Sade et al., 2010; Zhang et al., 2008a; Zhou et al., 2014). The reduced EL in FaPIP2; 1-transgenic plants under drought stress indicated that FaPIP2;1 positively contributed to the maintenance of cellular membrane stability. Limited information is available on AQP involvement in membrane stability under drought stress. It was reported that leaf EL was lower under chilling stress in rice overexpressing $O s P I P 2 ; 7$ compared with the controls ( $\mathrm{Li}$ et al., 2008 ) and plants overexpressing BjPIP1 showed lower levels of EL under cadmium stress (Zhang et al., 2008b). In addition, FaPIP2;1-transgenic plants were able to maintain greater levels of Chl and Pn, suggesting that overexpression of FaPIP 2;1 improved photosynthetic capacity under drought stress. The improvement in Pn could also be due to AQP regulation of stomatal movement, controlling water moving across the membranes (Yang et al., 2006). The positive physiological effects of FaPIP2;1 overexpression could contribute to the continued and increased leaf production and higher survival rates of plants during drought stress. However, overexpressing FaPIP2;1 did not have any impact on root growth in arabidopsis during this study. In some studies, AQP overexpression was found to promote root development under nonstress conditions, as well as drought, cold, and salinity stresses (Hu et al., 2012; Li et al., 2009; Xu et al., 2014; Zhou et al., 2012a). For example, transgenic tobacco with overexpression of $T a A Q P 7$ and TaAQP8 produced longer root length under drought and salinity stress, respectively (Hu et al., 2012; Zhou et al., 2012a). Transgenic arabidopsis plants overexpressing MaPIP1;1 exhibited increased primary root length with greater abundance of root hairs but fewer lateral roots than the WT seedlings (Xu et al., 2014). In this study, transgenic arabidopsis overexpressing FaPIP2; 1 did not display a morerobust root system than the WT but plants maintained higher leaf water status, and it could therefore be postulated that overexpressing FaPIP2;1 may promote root uptake efficiency by affecting root hydraulic conductivity (Yamauchi, 2004). However, the hypothesis of FaPIP2; 1 regulation of root water uptake deserves further testing and confirmation in future research. Nevertheless, our results suggest that FaPIP2;1 serves positive roles in plant drought tolerance mainly due to the alteration of leaf growth and physiological activities in arabidopsis.

\section{Conclusions}

An AQP gene of PIP2 subgroup, FaPIP2; 1, from tall fescue was cloned and characterized. FaPIP2; 1 overexpression conferred tolerance to drought stress in arabidopsis by enhancing leaf water status, $\mathrm{Chl}$, and $\mathrm{Pn}$, as well as maintaining improved cellular membrane stability relative to the WT plants. Future work will emphasize the regulatory mechanisms of FaPIP2; 1 conferring drought tolerance for leaves and roots. FaPIP2;1 could be a useful candidate gene transferred to tall fescue and other grass species for developing drought-tolerant germplasm.

\section{Literature Cited}

Aharon, R. 2003. Overexpression of a plasma membrane aquaporin in transgenic tobacco improves plant vigor under favorable growth conditions but not under drought or salt stress. Plant Cell 15:439-447.

Alexandersson, E., L. Fraysse, S. Sjovall-Larsen, S. Gustavsson, M. Fellert, M. Karlsson, U. Johanson, and P. Kjellbom. 2005. Whole gene family expression and drought stress regulation of aquaporins. Plant Mol. Biol. 59:469-484.

Arnon, D.I. 1949. Copper enzymes in isolated chloroplasts. Polyphenoloxidase in Beta vulgaris. Plant Physiol. 24:1-15.

Aroca, R., G. Amodeo, S. Fernandez-Illescas, E.M. Herman, F. Chaumont, and M.J. Chrispeels. 2005. The role of aquaporins and membrane damage in chilling and hydrogen peroxide induced changes in the hydraulic conductance of maize roots. Plant Physiol. 137:341-353. 
Barrs, H.D. and P.E. Weatherley. 1962. A re-examination of the relative turgidity technique for estimating water deficits in leaves. Austral. J. Biol. Sci. 15:413-428.

Blum, A. and A. Ebercon. 1981. Cell membrane stability as a measure of drought and heat tolerance in wheat. Crop Sci. $21: 43-47$.

Chaumont, F., F. Barrieu, E. Wojcik, M.J. Chrispeels, and R. Jung. 2001. Aquaporins constitute a large and highly divergent protein family in maize. Plant Physiol. 125:1206-1215.

Clough, S.J. and A.F. Bent. 1998. Floral dip: A simplified method for Agrobacterium-mediated transformation of Arabidopsis thaliana. Plant J. 16:735-743.

Cui, X.H., F.S. Hao, H. Chen, J. Chen, and X.C. Wang. 2008. Expression of the Vicia faba VfPIPl gene in Arabidopsis thaliana plants improves their drought resistance. J. Plant Res. 121:207-214.

DaCosta, M., Z. Wangand, and B. Huang. 2004. Physiological adaptation of kentucky bluegrass to localized soil drying. Crop Sci. 44:1307-1314.

Danielson, J.A. and U. Johanson. 2008. Unexpected complexity of the aquaporin gene family in the moss Physcomitrella patens. BMC Plant Biol. 8:45.

Diedhiou, C.J., O.V. Popovaand, and D. Golldack. 2009. Transcript profiling of the salt-tolerant Festuca rubra ssp. litoralis reveals a regulatory network controlling salt acclimatization. J. Plant Physiol. 166:697-711.

Fischer, R.A. and N.C. Turner. 1978. Plant productivity in the arid and semiarid zones. Annu. Rev. Plant Physiol. 29:277-317.

Forrest, K.L. and M. Bhave. 2007. Major intrinsic proteins (MIPs) in plants: A complex gene family with major impacts on plant phenotype. Funct. Integr. Genomics 7:263-289.

Fry, J. and B. Huang. 2004. Applied turfgrass science and physiology. Wiley, Hoboken, NJ.

Gao, Z., X. He, B. Zhao, C. Zhou, Y. Liang, R. Ge, Y. Shen, and Z. Huang. 2010. Overexpressing a putative aquaporin gene from wheat, TaNIP, enhances salt tolerance in transgenic Arabidopsis. Plant Cell Physiol. 51:767-775.

Gish, W. and D.J. States. 1993. Identification of protein coding regions by database similarity search. Nat. Genet. 3:266-272.

Gomes, D., A. Agasse, P. Thiébaud, S. Delrot, H. Gerós, and F. Chaumont. 2009. Aquaporins are multifunctional water and solute transporters highly divergent in living organisms. Biochimica et Biophysica Acta Biomembranes 1788:1213-1228.

Guo, L., Z.Y. Wang, H. Lin, W.E. Cui, J. Chen, M. Liu, Z.L. Chen, L.J. $\mathrm{Qu}$, and $\mathrm{H}$. Gu. 2006. Expression and functional analysis of the rice plasma-membrane intrinsic protein gene family. Cell Res. 16:277286.

Gupta, A.B. and R. Sankararamakrishnan. 2009. Genome-wide analysis of major intrinsic proteins in the tree plant Populus trichocarpa: Characterization of XIP subfamily of aquaporins from evolutionary perspective. BMC Plant Biol. 9:134.

Hall, T.A. 1999. Bioedit: A user-friendly biological sequence alignment editor and analysis program for Windows 95/98/N. Nucl. Acids Symp. Ser. 41:95-98.

Hanba, Y.T., M. Shibasaka, Y. Hayashi, T. Hayakawa, K. Kasamo, I. Terashima, and M. Katsuhara. 2004. Overexpression of the barley aquaporin HvPIP2;1 increases internal $\mathrm{CO}_{2}$ conductance and $\mathrm{CO}_{2}$ assimilation in the leaves of transgenic rice plants. Plant Cell Physiol. 45:521-529.

Heymann, J.B. and A. Engel. 1999. Aquaporins: Phylogeny, structure, and physiology of water channels. News Physiol. Sci. 14:187-193.

Hoagland, D.R. and D.I. Arnon. 1950. The water-culture method for growing plants without soil. 2nd ed. Circular 347. California Agr. Expt. Sta., Berkeley, CA.

Hu, W., Q. Yuan, Y. Wang, R. Cai, X. Deng, J. Wang, S. Zhou, M. Chen, L. Chen, C. Huang, Z. Ma, G. Yang, and G. He. 2012. Overexpression of a wheat aquaporin gene, TaAQP8, enhances salt stress tolerance in transgenic tobacco. Plant Cell Physiol. 53:21272141

Jang, J.Y., D.G. Kim, Y.O. Kim, J.S. Kim, and H. Kang. 2004. An expression analysis of a gene family encoding plasma membrane aquaporins in response to abiotic stresses in Arabidopsis thaliana. Plant Mol. Biol. 54:713-725.

Johanson, U., M. Karlsson, I. Johansson, S. Gustavsson, S. Sjovall, L. Fraysse, A.R. Weig, and P. Kjellbom. 2001. The complete set of genes encoding major intrinsic proteins in Arabidopsis provides a framework for a new nomenclature for major intrinsic proteins in plants. Plant Physiol. 126:1358-1369.

Li, D.D., Y.J. Wu, X.M. Ruan, B. Li, L. Zhu, H. Wang, and X.B. Li. 2009. Expressions of three cotton genes encoding the PIP proteins are regulated in root development and in response to stresses. Plant Cell Rpt. 28:291-300.

Li, G.W., M.H. Zhang, W.M. Cai, W.N. Sun, and W.A. Su. 2008. Characterization of OsPIP2; 7 , a water channel protein in rice. Plant Cell Physiol. 49:1851-1858.

Lian, H., X. Yu, Q. Ye, X. Ding, Y. Kitagawa, S. Kwak, W. Su, and Z. Tang. 2004. The role of aquaporin RWC3 in drought avoidance in rice. Plant Cell Physiol. 45:481-489.

Lian, H.L., X. Yu, D. Lane, W.N. Sun, Z.C. Tang, and W.A. Su. 2006. Upland rice and lowland rice exhibited different PIP expression under water deficit and ABA treatment. Cell Res. 16:651-660.

Mahdieh, M., A. Mostajeran, T. Horie, and M. Katsuhara. 2008. Drought stress alters water relations and expression of PIP-type aquaporin genes in Nicotiana tabacum plants. Plant Cell Physiol. 49:801-813.

Maurel, C. 2007. Plant aquaporins: Novel functions and regulation properties. FEBS Lett. 581:2227-2236.

Maurel, C., L. Verdoucq, D. Luu, and V. Santoni. 2008. Plant aquaporins: Membrane channels with multiple integrated functions. Annu. Rev. Plant Biol. 59:595-624.

Mian, M.A., Y. Zhang, Z.Y. Wang, J.Y. Zhang, X. Cheng, L. Chen, K. Chekhovskiy, X. Dai, C. Mao, F. Cheung, X. Zhao, J. He, A.D. Scott, C.D. Town, and G.D. May. 2008. Analysis of tall fescue ESTs representing different abiotic stresses, tissue types and developmental stages. BMC Plant Biol. 8:27.

Michel, B.E. 1983. Evaluation of the water potentials of solutions of polyethylene glycol 8000 both in the absence and presence of other solutes. Plant Physiol. 72:66-70.

Sade, N., M. Gebretsadik, R. Seligmann, A. Schwartz, R. Wallach, and M. Moshelion. 2010. The role of tobacco Aquaporin1 in improving water use efficiency, hydraulic conductivity, and yield production under salt stress. Plant Physiol. 152:245-254.

Sakurai, J., F. Ishikawa, T. Yamaguchi, M. Uemura, and M. Maeshima. 2005. Identification of 33 rice aquaporin genes and analysis of their expression and function. Plant Cell Physiol. 46:1568-1577.

Suga, S., S. Komatsuand, and M. Maeshima. 2002. Aquaporin isoforms responsive to salt and water stresses and phytohormones in radish seedlings. Plant Cell Physiol. 43:1229-1237.

Tamura, K., J. Dudley, M. Nei, and S. Kumar. 2007. MEGA4: Molecular evolutionary genetics analysis (MEGA) software version 4.0. Mol. Biol. Evol. 24:1596-1599.

Tyerman, S.D., C.M. Niemietzand, and H. Bramley. 2002. Plant aquaporins: Multifunctional water and solute channels with expanding roles. Plant Cell Environ. 25:173-194.

Vera-Estrella, R., B.J. Barkla, H.J. Bohnert, and O. Pantoja. 2004. Novel regulation of aquaporins during osmotic stress. Plant Physiol. $135: 2318-2329$

Wei, H., A.L. Dhanaraj, R. Arora, L.J. Rowland, Y. Fu, and L. Sunday. 2006. Identification of cold acclimation-responsive Rhododendron genes for lipid metabolism, membrane transport and lignin biosynthesis: Importance of moderately abundant ESTs in genomic studies. Plant Cell Environ. 29:558-570.

Xu, Y., W. Hu, J. Liu, J. Zhang, C. Jia, H. Miao, B. Xu, and Z. Jin. 2014. A banana aquaporin gene, MaPIP1; 1 , is involved in tolerance to drought and salt stresses. BMC Plant Biol. 14:59. 
Yamada, S., T. Komori, P.N. Myers, S. Kuwata, T. Kubo, and H. Imaseki. 1997. Expression of plasma membrane water channel genes under water stress in Nicotiana excelsior. Plant Cell Physiol. 38:1226-1231.

Yamauchi, A. 2004. Root system, p. 668-675. In: Encyclopedia of agricultural sciences [in Japanese]. Yokendo, Tokyo, Japan.

Yamaguchi-Shinozaki, K., M. Koizumi, S. Urao, and K. Shinozaki. 1992. Molecular cloning and characterization of $9 \mathrm{cDNAs}$ for genes that are responsive to desiccation in Arabidopsis thaliana: Sequence analysis of one cDNA clone that encodes a putative transmembrane channel protein. Plant Cell Physiol. 33:217-224.

Yang, H., X. Zhang, X. Wang, and J. Zhang. 2006. Water channels are involved in stomatal oscillations encoded by parameter-specific cytosolic calcium oscillations. J. Integr. Plant Biol. 48:790-799.

Yu, X., Y.H. Peng, M.H. Zhang, Y.J. Shao, W.A. Su, and Z.C. Tang. 2006. Water relations and an expression analysis of plasma membrane intrinsic proteins in sensitive and tolerant rice during chilling and recovery. Cell Res. 16:599-608.
Zhang, J., Z. Deng, S. Cao, X. Wang, A. Zhang, and X. Zhang. 2008a. Isolation of six novel aquaporin genes from Triticum aestivum $\mathrm{L}$. and functional analysis of TaAQP6 in water redistribution. Plant Mol. Biol. Rpt. 26:32-45.

Zhang, Y., Z. Wang, T. Chai, Z. Wen, and H. Zhang. 2008b. Indian mustard aquaporin improves drought and heavy-metal resistance in tobacco. Mol. Biotechnol. 40:280-292.

Zhou, L., C. Wang, R. Liu, Q. Han, R.K. Vandeleur, J. Du, S. Tyerman, and H. Shou. 2014. Constitutive overexpression of soybean plasma membrane intrinsic protein GmPIP1;6 confers salt tolerance. BMC Plant Biol. 14:181.

Zhou, S., W. Hu, X. Deng, Z. Ma, L. Chen, C. Huang, C. Wang, J. Wang, Y. He, G. Yang, and G. He. 2012a. Overexpression of the wheat aquaporin gene, TaAQP7, enhances drought tolerance in transgenic tobacco. PLoS One 7(12):e52439.

Zhou, Y., C.J. Lambrides, R. Kearns, C. Ye, and S. Fukai. 2012b. Water use, water use efficiency and drought resistance among warm-season turfgrasses in shallow soil profiles. Funct. Plant Biol. 39:116-125. 